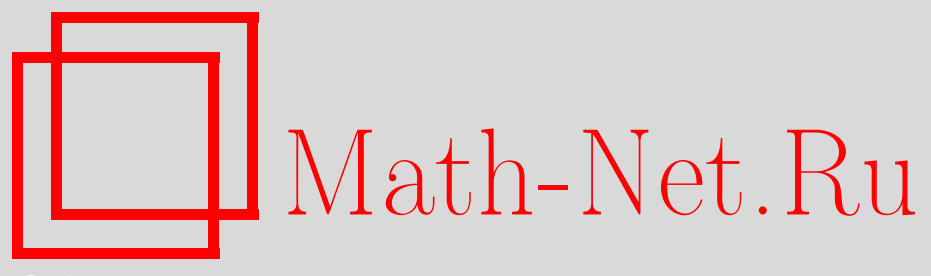

А. П. Исаев, Функциональные уравнения для трансферматричных операторов в моделях открытых цепочек Гекке, ТМФ, 2007, том 150, номер 2, 219-236

DOI: https://doi.org/10.4213/tmf5975

Использование Общероссийского математического портала Math-Net.Ru подразумевает, что вы прочитали и согласны с пользовательским соглашением http://www . mathnet.ru/rus/agreement

Параметры загрузки:

IP : 54.197 .130 .99

26 апреля 2023 г., 04:29:48

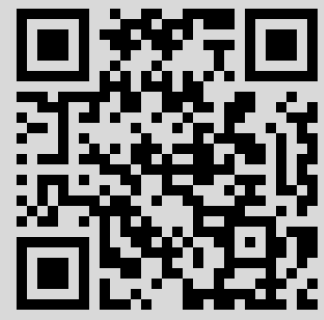




\title{
2007 г.
}

\author{
А. П. Исаев*
}

\section{ФУНКЦИОНАЛЬНЫЕ УРАВНЕНИЯ ДЛЯ ТРАНСФЕР-МАТРИЧНЫХ ОПЕРАТОРОВ В МОДЕЛЯХ ОТКРЫТЫХ ЦЕПОЧЕК ГЕККЕ}

Рассмотрены интегрируемые модели открытых цепочек, сформулированные в терминах образующих аффинных алгебр Гекке. С помощью fusion-процедуры (процедуры слияния) построена иерархия коммутирующих элементов, которые являются аналогами коммутативных транфер-матриц. Эти элементы удовлетворяют набору функциональных соотношений, которые обобщают функциональные соотношения для семейства трансфер-матриц в решаемых моделях спиновых цепочек $U_{q}(g l(n \mid m))$-типа.

Ключевые слова: интегрируемые модели спиновых цепочек, аффинная алгебра Гекке, алгебра Темперли-Либа, трансфер-матрица.

\section{1. ВВЕДЕНИЕ}

В нашей недавней работе [1] были найдены неполиномиальные бакстеризованные решения уравнения отражения, ассоциированного с аффинными алгебрами Гекке и аффинными алгебрами Бирмана-Мураками-Венцеля. Из этих решений в случае алгебры Гекке можно получить (детали изложены в [1]) полиномиальные решения, предложенные в [2]. В [1] также обсуждались связи с интегрируемыми моделями на цепочках с нетривиальными граничными условиями.

В данной работе мы сконцентрируемся на исследовании моделей, сформулированных в терминах образующих аффинной алгебры Гекке. Пусть сплетающие элементы $\left\{\sigma_{1}, \ldots, \sigma_{M}\right\}$ и аффинный элемент $y_{1}$ являются образующими аффинной алгебры Гекке $\widehat{H}_{M+1}(q)$ (определение алгебры $\widehat{H}_{M+1}(q)$ дано в следующем разделе). В работе [1] мы предложили рассматривать модели на открытой цепочке с гамильтонианом

$$
\mathcal{H}_{M}=\sum_{k=1}^{M} \sigma_{k}+\left(q-q^{-1}\right) \frac{\xi}{y_{1}-\xi},
$$

где $q$ - параметр алгебры $\widehat{H}_{M+1}(q)$, а $\xi$ - параметр модели, который определяет граничное условие и возникает в данном гамильтониане в результате использования

* Объединенный институт ядерных исследований, Дубна, Московская обл., Россия. E-mail: isaevap@theor.jinr.ru 
неполиномиального решения [1]

$$
y_{1}(x)=\frac{y_{1}-\xi x}{y_{1}-\xi x^{-1}}
$$

(здесь $x$ - спектральный параметр) уравнения отражения, ассоциированного с аффинной алгеброй Гекке. Модель с гамильтонианом (1) обобщает $X X Z$-модель на открытой спиновой цепочке. Действительно, как было показано в [1], эта модель описывает единым образом целый набор интегрируемых систем для любого представления алгебры $\widehat{H}_{M+1}(q)$. Наиболее интересны (с точки зрения приложений) так называемые $R$-матричные представления $\widehat{H}_{M+1}(q)$. Известно, что алгебра Гекке может быть реализована с помощью $R$-матриц в фундаментальном представлении (фундаментальных $R$-матриц) для случая $U_{q}(g l(n))$ [3], [4] и для случая $U_{q}(g l(n \mid m))$ [5]. В случае $U_{q}(g l(n \mid m))$ эта реализация имеет вид

$$
\rho\left(\sigma_{i}\right)=I^{\otimes(i-1)} \otimes \widehat{R} \otimes I^{\otimes(M-i)}=: \widehat{R}_{i i+1},
$$

где $I \in \operatorname{Mat}(n+m)$ - единичная матрица и $\widehat{R}$ - фундаментальная $R$-матрица для $U_{q}(g l(n \mid m))$, записанная в форме образующей группы кос. Для аффинной образующей мы имеем

$$
\rho\left(y_{1}\right)=L \otimes I^{\otimes M}=: L_{1},
$$

где элементы матрицы $L \in \operatorname{Mat}(n+m)$ являются генераторами алгебры уравнения отражения $U_{q}(g l(n \mid m))$-типа $\widehat{R}_{12} L_{1} \widehat{R}_{12} L_{1}=L_{1} \widehat{R}_{12} L_{1} \widehat{R}_{12}$. Все эти $R$-матричные представления $\rho$ ведут к интегрируемым системам на цепочках с гамильтонианами $\rho\left(\mathcal{H}_{M}\right)$. Рассматривая для алгебры $\widehat{H}_{M+1}$ специальное представление $\rho$, соответствующее $R$-матрице $U_{q}(g l(2))$-типа, мы воспроизводим из (1) гамильтониан $X X Z$ модели открытой спиновой цепочки. В дальнейшем мы называем модель с гамильтонианом (1), построенную в терминах образующих алгебры Гекке $\widehat{H}_{M+1}$, моделью открытой цепочки Гекке.

В данной статье для модели открытой цепочки Гекке с помощью алгебраического аналога fusion-процедуры [6], [7] построена иерархия коммутирующих элементов (аналогов трансфер-матриц). Оказывается, что эти элементы удовлетворяют функциональным соотношениям, которые обобщают функциональные соотношения для трансфер-матриц в решаемых моделях на открытых спиновых цепочках (см., например, [8] и ссылки, приведенные в этих работах).

Структура статьи следующая. В разделе 2 мы даем определение аффинной алгебры Гекке и напоминаем алгебраическую формулировку [1] системы открытой цепочки Гекке. В частности, мы исследуем коммутирующие элементы трансферматричного типа, которые являются производящими функциями гамильтонианов для рассматриваемой модели. В разделе 3 представлено алгебраическое описание fusion-процедуры для решений уравнения отражения и для соответствующих коммутирующих элементов, аналогичных трансфер-матрицам. В этом разделе мы выводим функциональные соотношения для таких коммутирующих элементов в модели открытой цепочки Гекке. В разделах 4 и 5 мы обсуждаем фактор Темперли-Либа 
для алгебры Гекке и формулируем соответствующую модель цепочки Гекке, в которой удается получить замкнутые функциональные уравнения для элементов типа трансфер-матриц. Заметим, что наш анализ связан с подходом, который предложен в [9] для исследования систем спиновых цепочек, построенных с использованием $R$-матриц, реализующих алгебру Темперли-Либа. В заключении мы кратко обсуждаем взаимосвязи между алгебраическим подходом этой статьи и стандартной техникой уравнений отражения, развитой в [10] для исследования систем открытых цепочек.

\section{2. ОТКРЫТЫЕ ЦЕПОЧКИ ГЕККЕ}

Группа кос $\mathcal{B}_{M+1}$ генерируется элементами $\sigma_{i}, i=1, \ldots, M$, которые удовлетворяют соотношениям

$$
\sigma_{i} \sigma_{i+1} \sigma_{i}=\sigma_{i+1} \sigma_{i} \sigma_{i+1}, \quad \sigma_{i} \sigma_{j}=\sigma_{j} \sigma_{i} \quad \text { для } \quad|i-j|>1 .
$$

Алгебра Гекке $A$-типа $H_{M+1}(q)$ является фактором групповой алгебры группы кос $\mathcal{B}_{M+1}$ по дополнительным соотношениям Гекке

$$
\sigma_{i}^{2}-1=\lambda \sigma_{i} \quad(i=1, \ldots, M)
$$

где $\lambda:=\left(q-q^{-1}\right)$ и $q \in \mathbb{C} \backslash\{0\}-$ параметр алгебры. Пусть $x \in \mathbb{C}$ - спектральный параметр. Определим бакстеризованные элементы [3] (см. также [5], [11])

$$
\sigma_{n}(x):=\sigma_{n}-x \sigma_{n}^{-1} \in H_{M+1}(q),
$$

которые являются решениями уравнения Янга-Бакстера

$$
\sigma_{n}(x) \sigma_{n-1}(x y) \sigma_{n}(y)=\sigma_{n-1}(y) \sigma_{n}(x y) \sigma_{n-1}(x)
$$

и удовлетворяют соотношению $\sigma_{n}(x) \sigma_{n}(y)=\lambda \sigma_{n}(x y)+(1-x)(1-y)$. Нормализованные элементы [1]

$$
e_{n}^{-}(x):=\frac{\sigma_{n}-x \sigma_{n}^{-1}}{q x-q^{-1}}, \quad e_{n}^{+}(x):=\frac{\sigma_{n}-x \sigma_{n}^{-1}}{q-q^{-1} x}
$$

подчиняются условиям унитарности $e_{n}^{+}(x) e_{n}^{+}\left(x^{-1}\right)=1, e_{n}^{-}(x) e_{n}^{-}\left(x^{-1}\right)=1$. Эти нормализованные элементы полезны для определения симметризаторов $A_{1 \rightarrow n}^{+}$и антисимметризаторов $A_{1 \rightarrow n}^{-}(n=1,2, \ldots, M+1)$ для алгебры Гекке $H_{M+1}:=H_{M+1}(q)$. Операторы $A_{1 \rightarrow n}^{ \pm}$определяются индуктивно с помощью рекуррентных соотношений [3] (см. также [11] и ссылки, приведенные там)

$$
A_{1 \rightarrow n+1}^{ \pm}=A_{1 \rightarrow n}^{ \pm} e_{n}^{ \pm}\left(q^{\mp 2 n}\right) A_{1 \rightarrow n}^{ \pm}, \quad A_{1 \rightarrow 1}^{ \pm}:=1
$$

и удовлетворяют равенствам

$$
\begin{aligned}
\sigma_{i} A_{1 \rightarrow n}^{ \pm} & =A_{1 \rightarrow n}^{ \pm} \sigma_{i}= \pm q^{ \pm 1} A_{1 \rightarrow n}^{ \pm} \quad(i=1, \ldots, n-1), \\
A_{1 \rightarrow n}^{ \pm} A_{1 \rightarrow m}^{ \pm} & =A_{1 \rightarrow n}^{ \pm}=A_{1 \rightarrow m}^{ \pm} A_{1 \rightarrow n}^{ \pm} \quad(n \geqslant m) ; \quad A_{1 \rightarrow n}^{ \pm} A_{1 \rightarrow m}^{\mp}=0, \\
e_{i}^{ \pm}(x) A_{1 \rightarrow n}^{ \pm} & =A_{1 \rightarrow n}^{ \pm} e_{i}^{ \pm}(x)=A_{1 \rightarrow n}^{ \pm} \quad(i=1, \ldots, n-1) .
\end{aligned}
$$


Аффинная алгебра Гекке $\widehat{H}_{M+1}$ (см., например, главу 12.3 в книге [12]) есть расширение алгебры Гекке $H_{M+1}$. Алгебра $\widehat{H}_{M+1}$ генерируется элементами $\sigma_{i}(i=$ $1, \ldots, M)$ алгебры $H_{M+1}$ и аффинными образующими $y_{k}(k=1, \ldots, M+1)$, которые удовлетворяют соотношениям

$$
y_{k+1}=\sigma_{k} y_{k} \sigma_{k}, \quad y_{k} y_{j}=y_{j} y_{k}, \quad y_{j} \sigma_{i}=\sigma_{i} y_{j} \quad(j \neq i, i+1) .
$$

Элементы $\left\{y_{k}\right\}$ формируют коммутативную подалгебру в $\widehat{H}_{M+1}$, в то время как симметрические функции от переменных $y_{k}$ центральны в алгебре $\widehat{H}_{M+1}$.

С помощью индукции можно доказать, что элемент

$$
y_{n}(x)=\sigma_{n-1}\left(\frac{x}{\xi_{n-1}}\right) \ldots \sigma_{2}\left(\frac{x}{\xi_{2}}\right) \sigma_{1}\left(\frac{x}{\xi_{1}}\right) y_{1}(x) \sigma_{1}\left(x \xi_{1}\right) \sigma_{2}\left(x \xi_{2}\right) \ldots \sigma_{n-1}\left(x \xi_{n-1}\right)
$$

является решением (для любого $\left.\xi_{1}, \ldots, \xi_{n-1} \in \mathbb{C} \backslash\{0\}\right)$ уравнения отражения

$$
\sigma_{n}\left(x z^{-1}\right) y_{n}(x) \sigma_{n}(x z) y_{n}(z)=y_{n}(z) \sigma_{n}(x z) y_{n}(x) \sigma_{n}\left(x z^{-1}\right)
$$

где $y_{1}(x) \in \widehat{H}_{M+1}$ - любое локальное (т.е. $\left[y_{1}(x), \sigma_{k}\right]=0$ при $k>1$ ) решение уравнения (11) для $n=1$. В работе [1] мы нашли такое локальное решение, которое является рациональной функцией переменной $y_{1}$ :

$$
y_{1}(x)=\frac{y_{1}-\xi x}{y_{1}-\xi x^{-1}}
$$

где $y_{1}$ - аффинная образующая алгебры $\widehat{H}_{M+1}$ и $\xi \in \mathbb{C}$ - произвольный параметр. Оператор $y_{1}(x)(12)$ является регулярным: $y_{1}(1)=1$, и подчиняется условию унитарности $y_{1}(x) y_{1}\left(x^{-1}\right)=1$. Ниже мы рассматриваем специальную форму элемента (10) алгебры Гекке, когда все $\xi_{k}=1$ :

$$
y_{n}(x)=\sigma_{n-1}(x) \ldots \sigma_{2}(x) \sigma_{1}(x) y_{1}(x) \sigma_{1}(x) \sigma_{2}(x) \ldots \sigma_{n-1}(x) .
$$

Этот элемент является алгебраическим аналогом матрицы монодромии Склянина [10]. Он был использован в [1] для построения интегрируемых систем на цепочках.

Рассмотрим следующее естественное включение подалгебр $\widehat{H}_{1} \subset \widehat{H}_{2} \subset \ldots$ $\cdots \subset \widehat{H}_{M+1}$ :

$$
\left\{y_{1} ; \sigma_{1}, \ldots, \sigma_{n-1}\right\}=\widehat{H}_{n} \subset \widehat{H}_{n+1}=\left\{y_{1} ; \sigma_{1}, \ldots, \sigma_{n-1}, \sigma_{n}\right\}
$$

Далее, следуя работе [1], мы оснащаем алгебру $\widehat{H}_{M+1}$ линейными отображениями

$$
\operatorname{Tr}_{D(n+1)}: \widehat{H}_{n+1} \rightarrow \widehat{H}_{n}
$$

из алгебр $\widehat{H}_{n+1}$ в их подалгебры $\widehat{H}_{n}$, причем эти отображения таковы, что для всех $X, X^{\prime} \in \widehat{H}_{n}$ и $Y \in \widehat{H}_{n+1}$

$$
\begin{aligned}
& \operatorname{Tr}_{D(n+1)}(X)=D^{(0)} X, \quad \operatorname{Tr}_{D(n+1)}\left(X Y X^{\prime}\right)=X \operatorname{Tr}_{D(n+1)}(Y) X^{\prime}, \\
& \operatorname{Tr}_{D(n+1)}\left(\sigma_{n}^{ \pm 1} X \sigma_{n}^{\mp 1}\right)=\operatorname{Tr}_{D(n)}(X), \quad \operatorname{Tr}_{D(n+1)}\left(X \sigma_{n} X^{\prime}\right)=X X^{\prime}, \\
& \operatorname{Tr}_{D(1)}\left(y_{1}^{k}\right)=D^{(k)}, \quad \operatorname{Tr}_{D(n)} \operatorname{Tr}_{D(n+1)}\left(\sigma_{n} Y\right)=\operatorname{Tr}_{D(n)} \operatorname{Tr}_{D(n+1)}\left(Y \sigma_{n}\right),
\end{aligned}
$$


где $D^{(k)} \in \mathbb{C} \backslash\{0\}(k \in \mathbb{Z})$ являются константами. Подчеркнем, что объекты $D^{(k)}$ могли бы рассматриваться как добавочные образующие абелевой подалгебры $\widehat{H}_{0}$, которая расширяет $\widehat{H}_{M+1}$ и является центральной в $\widehat{H}_{M+1}$. Однако для дальнейших рассуждений нам достаточно считать $D^{(k)}$ константами.

Используя отображения $\operatorname{Tr}_{D(n+1)}$, мы можем показать, что бакстеризованные элементы $\sigma_{n}(x)(4)$ подчиняются следующему тождеству (для всех $X \in \widehat{H}_{n}$ и любых $x, z)$ :

$$
\operatorname{Tr}_{D(n+1)}\left(\sigma_{n}(x) X \sigma_{n}(z)\right)=(1-x)(1-z) \operatorname{Tr}_{D(n)}(X)+\lambda\left(1-\frac{x z}{b}\right) X
$$

где

$$
b:=\frac{1}{1-\lambda D^{(0)}} .
$$

Применяя это тождество к уравнению отражения (11), мы получаем

$$
\left[\tau_{n-1}(x), y_{n}(z)\right]=\frac{\lambda\left(x b^{-1}-x^{-1}\right)}{\left(\left(x+x^{-1}\right)-\left(z+z^{-1}\right)\right)}\left[y_{n}(x), y_{n}(z)\right]
$$

где операторы

$$
\tau_{n}(x)=\operatorname{Tr}_{D(n+1)}\left(y_{n+1}(x)\right)
$$

образуют коммутативное семейство, $\left[\tau_{n}(x), \tau_{n}(z)\right]=0$ (для любых $\left.x, z\right)$ [1].

Теперь мы сформулируем модели открытых цепочек Гекке, используя алгебраические аналоги трансфер-матриц Склянина [10]. В нашем случае такие аналоги (трансфер-матричные операторы $\left.\tau_{n}(x)\right)$ являются элементами алгебры $\widehat{H}_{n}$ и представляются в виде $(16)$, где решение $y_{n+1}(x)$ уравнения отражения выбрано в специальном виде (13):

$$
\tau_{n}(x)=\operatorname{Tr}_{D(n+1)}\left(y_{n+1}(x)\right)=\operatorname{Tr}_{D(n+1)}\left(\sigma_{n}(x) \ldots \sigma_{1}(x) y_{1}(x) \sigma_{1}(x) \ldots \sigma_{n}(x)\right),
$$

а $y_{1}(x)$ является любым локальным и регулярным решением уравнения (11) для $n=$ 1 (например, можно взять решение (12)). Локальный гамильтониан для открытой цепочки Гекке имеет вид

$$
\mathcal{H}_{n}=\sum_{m=1}^{n-1} \sigma_{m}-\frac{\lambda}{2} y_{1}^{\prime}(1) .
$$

Этот гамильтониан (с точностью до нормировочной и аддитивной констант) может быть получен дифференцированием $\tau_{n}(x)$ по $x$ в точке $x=1$. Гамильтониан (18) описывает модель открытой цепочки с нетривиальным граничным условием на первом узле цепочки (которое задается вторым членом в (18)) и свободным граничным условием на последнем узле. Гамильтониан (1) получается подстановкой решения (12) в (18).

Из (15) следует, что трансфер-матричный оператор $\tau_{n}(x)(17)$ удовлетворяет рекуррентному уравнению

$$
\tau_{n}(x)=\lambda\left(1-\frac{x^{2}}{b}\right) y_{n}(x)+(1-x)^{2} \tau_{n-1}(x),
$$


которое имеет решение вида

$$
\tau_{n}(x)=\lambda\left(1-\frac{x^{2}}{b}\right)\left(\sum_{k=0}^{n-1}(1-x)^{2 k} y_{n-k}(x)\right)+(1-x)^{2 n} \operatorname{Tr}_{D(1)}\left(y_{1}(x)\right),
$$

где элементы монодромии $y_{k}(x)(13)$ являются решениями уравнения отражения (11) для $n=k$.

Рассмотрим модель открытой цепочки Гекке со свободными граничными условиями на обоих концах цепочки, т.е. при условии $y_{1}(x)=1$. В этом случае из $(20)$ мы выводим, что

$$
\begin{aligned}
\left.\tau_{n}(x)\right|_{y_{1}(x)=1} & =\operatorname{Tr}_{D(n+1)}\left(\sigma_{n}(x) \ldots \sigma_{2}(x) \sigma_{1}^{2}(x) \sigma_{2}(x) \ldots \sigma_{n}(x)\right)= \\
& =\lambda\left(1-\frac{x^{2}}{b}\right) J_{n}(x)+(1-x)^{2 n} D^{(0)}=: T_{n}^{(1)}(x),
\end{aligned}
$$

где $J_{n}(x)$ - специальный полином от спектрального параметра $x$ порядка $2 n-2$,

$$
\begin{aligned}
J_{n}(x) & =\left(\sum_{k=0}^{n-2}(1-x)^{2 k} \sigma_{n-k-1}(x) \ldots \sigma_{1}^{2}(x) \ldots \sigma_{n-k-1}(x)\right)+(1-x)^{2(n-1)}= \\
& =\lambda(1+x)\left(\sum_{a=1}^{2 n-3}(1-x)^{a}(\lambda x)^{2 n-3-a} j_{a}\right)+f_{n}(x),
\end{aligned}
$$

и $f_{n}(x)$ - скалярные полиномиальные функции от $x$,

$$
f_{n}(x)=\sum_{k=0}^{n-2}(1-x)^{2 k}\left((1-x)^{2}+\lambda^{2} x^{2}\right)^{n-k-1}+(1-x)^{2(n-1)} .
$$

Представление (22) следует из уравнений

$$
\sigma_{k}(x)=(1-x) \sigma_{k}+\lambda x, \quad \sigma_{k}^{2}(x)=\lambda(1+x)(1-x) \sigma_{k}+\left[(1-x)^{2}+\lambda^{2} x^{2}\right] .
$$

Так как элементы трансфер-матричного типа $\tau_{n}(x)$ образуют коммутативное семейство, $\left[\tau_{n}(x), \tau_{n}(z)\right]=0$, то мы получаем из $(21)$, (22) набор $\left\{j_{1}, j_{2}, \ldots, j_{2 n-3}\right\}$ из $2 n-3$ коммутативных элементов для алгебры Гекке $H_{n}$. Ясно, что

$$
\begin{aligned}
J_{n}(0) & =\sigma_{n-1} \ldots \sigma_{1}^{2} \ldots \sigma_{n-1}+\sigma_{n-2} \ldots \sigma_{1}^{2} \ldots \sigma_{n-2}+\cdots+\sigma_{1}^{2}+1= \\
& =\lambda \sum_{m=1}^{n-1} \sum_{k=1}^{m}\left(\sigma_{k} \ldots \sigma_{m-1} \sigma_{m} \sigma_{m-1} \ldots \sigma_{k}\right)+n=\lambda j_{2 n-3}+f_{n}(0),
\end{aligned}
$$

и, соответственно, старший оператор $j_{2 n-3}-$ центральный элемент в $H_{n}$. С другой стороны, мы имеем

$$
J_{n}^{\prime}(1)=-2 \lambda^{2 n-3}\left(\sum_{m=1}^{n-1} \sigma_{m}\right)+2 \lambda^{2 n-2}(n-1)=-2 \lambda^{2 n-3} j_{1}+f_{n}^{\prime}(1)
$$


и в соответствии с (18) получаем, что первый коэффициент в разложении (22),

$$
j_{1}=\mathcal{H}_{n}^{(\text {free })}=\sum_{m=1}^{n-1} \sigma_{m},
$$

равен гамильтониану для открытой цепочки Гекке со свободными концами.

Для первого нетривиального случая $n=3$ мы имеем набор коммутирующих элементов

$$
j_{1}=\sigma_{1}+\sigma_{2}, \quad j_{2}=\sigma_{1} \sigma_{2}+\sigma_{2} \sigma_{1}, \quad j_{3}=\sigma_{2} \sigma_{1} \sigma_{2}+\sigma_{1}+\sigma_{2} .
$$

Элемент $j_{1}$ совпадает с гамильтонианом $\mathcal{H}_{3}^{(\text {free) }}$ для открытой цепочки Гекке, а $j_{3}$ - центральный элемент в $H_{3}$. Характеристическое тождество для гамильтониана $j_{1}=\mathcal{H}_{3}^{(\text {free })}$ находится непосредственно:

$$
\left(j_{1}+2 q^{-1}\right)\left(j_{1}-2 q\right)\left(j_{1}-\lambda-1\right)\left(j_{1}-\lambda+1\right)=0
$$

оно означает, что $\operatorname{Spec}\left(\mathcal{H}_{3}^{(\text {free })}\right)=\left\{2 q,-2 q^{-1}, \lambda \pm 1\right\}$. Два первых собственных значения $\pm 2 q^{ \pm 1}$ соответствуют одномерным представлениям $\sigma_{i}= \pm q^{ \pm 1}(i=1,2)$ алгебры $H_{3}$. Дублет $\lambda \pm 1$ соответствует двумерному неприводимому представлению $H_{3}$ (по поводу теории представлений алгебр Гекке см. [11], [13] и ссылки, приведенные в этих работах).

Для случая $n=4$ мы получаем следующий набор коммутирующих элементов:

$$
\begin{aligned}
& j_{1}=\sum_{i=1}^{3} \sigma_{i}, \quad j_{2}=\left\{\sigma_{1}, \sigma_{2}\right\}_{+}+\left\{\sigma_{2}, \sigma_{3}\right\}_{+}+2 \sigma_{3} \sigma_{1}, \\
& j_{3}=\left\{\sigma_{1} \sigma_{3}, \sigma_{2}\right\}_{+}+\left(\sigma_{1}+\sigma_{3}\right) \sigma_{2}\left(\sigma_{1}+\sigma_{3}\right)+\lambda \sigma_{3} \sigma_{1}+2 \sum_{i=1}^{3} \sigma_{i}, \\
& j_{4}=\left\{\sigma_{2} \sigma_{3} \sigma_{2}, \sigma_{1}\right\}_{+}+\left\{\sigma_{2} \sigma_{1} \sigma_{2}, \sigma_{3}\right\}_{+}+\left\{\sigma_{2}, \sigma_{3}\right\}_{+}+\left\{\sigma_{2}, \sigma_{1}\right\}_{+}, \\
& j_{5}=\sigma_{1} \sigma_{2} \sigma_{3} \sigma_{2} \sigma_{1}+\sigma_{2} \sigma_{3} \sigma_{2}+\sigma_{1} \sigma_{2} \sigma_{1}+\sigma_{1}+\sigma_{2}+\sigma_{3},
\end{aligned}
$$

Коэффициент $j_{5}$ является центральным элементом в $H_{4}$. Заметим, что наиболее длинный элемент в $H_{4}$ вида $j=\sigma_{1} \sigma_{2} \sigma_{3} \sigma_{1} \sigma_{2} \sigma_{1}=\left(j_{5}-j_{1}\right)\left(j_{1}-\lambda\right)-j_{4}$ коммутирует с гамильтонианом $j_{1}=\mathcal{H}_{4}^{(\text {free) }}$. Спектр $\mathcal{H}_{4}^{(\text {free) }}$ состоит из следующих собственных значений: $\pm 3 q^{ \pm 1}$ для двух одномерных представлений, $2 q-q^{-1}, 2 q-q^{-1} \pm \sqrt{2}$ и $-2 q^{-1}+q,-2 q^{-1}+q \pm \sqrt{2}$ для двух дуальных друг к другу трехмерных неприводимых представлений и из собственных значений $\left(3 \lambda \pm \sqrt{q^{-2}+10+q^{2}}\right) / 2$ для двумерного неприводимого представления $H_{4}$.

ЗАмечаниЕ. Рассмотрим элемент $j$ в группе кос $B_{M+1}$ :

$$
j:=\left(\sigma_{1} \ldots \sigma_{M}\right)\left(\sigma_{1} \ldots \sigma_{M-1}\right) \ldots\left(\sigma_{1} \sigma_{2}\right) \cdot \sigma_{1} .
$$

Этот элемент коммутирует с элементом $\sum_{i=1}^{M} \sigma_{i}$ групповой алгебры группы кос $B_{M+1}$, так как $\sigma_{i} j=j \sigma_{M+1-i}$. Таким образом, для фактора Гекке $H_{M+1}$ групповой алгебры группы $B_{M+1}$ элемент $j \in H_{M+1}$ является сохраняющимся зарядом модели 
на цепочке с гамильтонианом $\mathcal{H}_{M+1}^{(\text {free) }}(23)$. Используя наиболее длинный элемент $j \in H_{n}$, мы можем показать, что операторы (21), (22) являются инвариантными по отношению к зеркальному преобразованию $\sigma_{k} \leftrightarrow \sigma_{n-k}$. Это демонстрирует зеркальную симметрию открытой цепочки Гекке с гамильтонианом (23) по отношению к взаимной замене первого и последнего узлов цепочки.

\section{FUSION-ПРОЦЕДУРА ДЛЯ БАКСТЕРИЗОВАННЫХ РЕШЕНИЙ УРАВНЕНИЯ ОТРАЖЕНИЯ}

Fusion-процедура для решений уравнения Янга-Бакстера была предложена в [6]. Fusion-процедура для решений уравнения отражения рассматривалась в [7], где она применялась для исследования $X X Z$-модели открытой спиновой цепочки. В этом разделе мы формулируем fusion-процедуру для решений уравнения отражения, записанных в терминах образующих аффинной алгебры Гекке. Ниже мы используем компактные обозначения

$$
e_{n}^{x}:=e_{n}^{+}(x), \quad y_{1}^{x}:=y_{1}(x)
$$

для бакстеризованных элементов $e_{n}^{+}(x)$, заданных в $(6)$, и решений $y_{1}(x)$ уравнения отражения (11).

Уравнение отражения (11) можно графически представить в виде
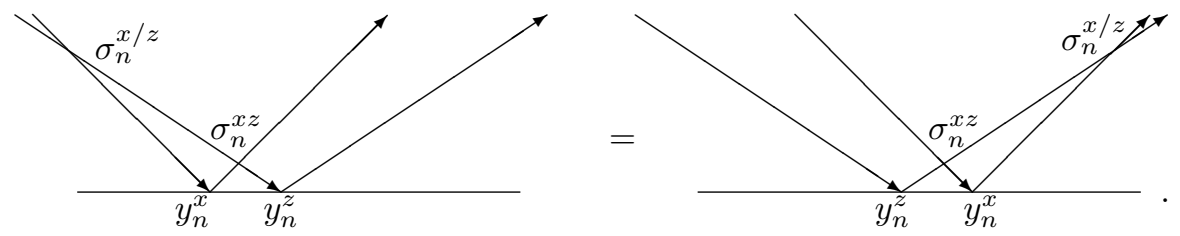

Рассмотрим последовательность изображений
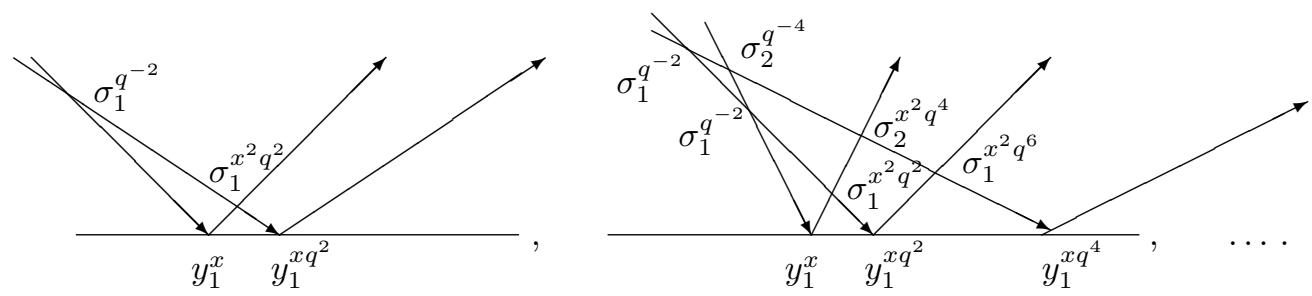

Используя эту последовательность, мы легко можем построить fusion-процедуру для решений уравнения отражения. Действительно, алгебраические выражения для 
этих диаграмм записываются как

$$
\begin{aligned}
Y_{1 \rightarrow 2}(x) & =e_{1}^{q^{-2}} \cdot\left[y_{1}^{x} e_{1}^{x^{2} q^{2}} y_{1}^{x q^{2}}\right]=e_{1}^{q^{-2}} \cdot y_{1 \rightarrow 2}(x), \\
Y_{1 \rightarrow 3}(x) & =\left[e_{1}^{q^{-2}} e_{2}^{q^{-4}} e_{1}^{q^{-2}}\right] \cdot\left[y_{1}^{x} e_{1}^{x^{2} q^{2}} y_{1}^{x q^{2}} e_{2}^{x^{2} q^{4}} e_{1}^{x^{2} q^{6}} y_{1}^{x q^{4}}\right]= \\
& =\left[e_{1}^{q^{-2}} e_{2}^{q^{-4}} e_{1}^{q^{-2}}\right] \cdot y_{1 \rightarrow 3}(x),
\end{aligned}
$$

где мы использовали обозначения (25). Формула, которая обобщает (27), имеет вид

$$
Y_{1 \rightarrow k}(x)=A_{1 \rightarrow k}^{+} \cdot y_{1 \rightarrow k}(x)=\bar{y}_{1 \rightarrow k}(x) \cdot A_{1 \rightarrow k}^{+},
$$

где

$$
\begin{aligned}
y_{1 \rightarrow k}(x):= & y_{1}^{x}\left[e_{1}^{x^{2} q^{2}} y_{1}^{x q^{2}}\right]\left[e_{2}^{x^{2} q^{4}} e_{1}^{x^{2} q^{6}} y_{1}^{x q^{4}}\right]\left[e_{3}^{x^{2} q^{6}} e_{2}^{x^{2} q^{8}} e_{1}^{x^{2} q^{10}} y_{1}^{x q^{6}}\right] \times \cdots \\
& \cdots \times\left[e_{k-1}^{x^{2} q^{2(k-1)}} e_{k-2}^{x^{2} q^{2 k}} e_{k-3}^{x^{2} q^{2(k+1)}} \ldots e_{2}^{x^{2} q^{2(2 k-4)}} e_{1}^{x^{2} q^{2(2 k-3)}} y_{1}^{x q^{2(k-1)}}\right]
\end{aligned}
$$

а $\bar{y}_{1 \rightarrow k}(x)$ получается из выражения $y_{1 \rightarrow k}(x)$, если его переписать в обратном порядке, справа налево; второе равенство в (28) получается с помощью уравнения Янга-Бакстера (5) и уравнения отражения (11) и может быть мгновенно воспроизведено, если рассмотреть графические представления (аналогичные тем, которые изображены в (26)) для обеих его частей. Соответствующее слияние (fusion) для бакстеризованных элементов дается выражением

$$
\begin{aligned}
\sigma_{1 \rightarrow k, k+1 \rightarrow 2 k}(x) & =A_{1 \rightarrow k}^{+} \cdot A_{k+1 \rightarrow 2 k}^{+} \cdot \sigma_{k+1 \leftarrow 1}^{x} \sigma_{k+2 \leftarrow 2}^{x q^{2}} \ldots \sigma_{2 k \leftarrow k}^{x q^{2(k-1)}} \equiv \\
& \equiv A_{1 \rightarrow k}^{+} \cdot A_{k+1 \rightarrow 2 k}^{+} \cdot \sigma_{k \rightarrow 2 k}^{x q^{-2(k-1)}} \ldots \sigma_{2 \rightarrow k+2}^{x q^{-2}} \sigma_{1 \rightarrow k+1}^{x}
\end{aligned}
$$

где

$$
\sigma_{k+1 \leftarrow 1}^{x}=e_{k}^{x q^{-2(k-1)}} \ldots e_{2}^{x q^{-2}} e_{1}^{x}, \quad \sigma_{1 \rightarrow k+1}^{x}=e_{1}^{x} e_{2}^{x q^{2}} \ldots e_{k}^{x q^{2(k-1)}} .
$$

Элементы (29) являются решениями высших уравнений Янга-Бакстера. Мы можем непосредственно проверить (например, используя графическое представление типа (26)), что (28) и (29) удовлетворяют уравнению

$$
\begin{aligned}
& \sigma_{1 \rightarrow k, k+1 \rightarrow 2 k}\left(x z^{-1}\right) Y_{1 \rightarrow k}(x) \sigma_{1 \rightarrow k, k+1 \rightarrow 2 k}(x z) Y_{1 \rightarrow k}(z)= \\
& \quad=Y_{1 \rightarrow k}(z) \sigma_{1 \rightarrow k, k+1 \rightarrow 2 k}(x z) Y_{1 \rightarrow k}(x) \sigma_{1 \rightarrow k, k+1 \rightarrow 2 k}\left(x z^{-1}\right),
\end{aligned}
$$

которое есть fusion-версия уравнения отражения (11).

Высшие элементы трансфер-матричного типа $\tau^{(k)}(x)$, которые соответствуют fusion-решению (28) уравнения отражения (30), имеют вид

$$
\tau^{(k)}(x)=\operatorname{Tr}_{D(1 \rightarrow k)}\left(Y_{1 \rightarrow k}(x)\right)=\operatorname{Tr}_{D(1 \rightarrow k)}\left(A_{1 \rightarrow k}^{+} \cdot y_{1 \rightarrow k}(x)\right) .
$$

В частности, для $k=1,2$ мы имеем

$$
\tau^{(1)}(x)=\operatorname{Tr}_{D(1)}\left(y_{1}^{x}\right), \quad \tau^{(2)}(x)=\operatorname{Tr}_{D(1 \rightarrow 2)}\left(e_{1}^{q^{-2}} y_{1}^{x}\left[e_{1}^{x^{2} q^{2}} y_{1}^{x q^{2}}\right]\right) .
$$

Ниже нам понадобится следующая лемма. 
Лемма. В алгебре Гекке справедливо следующее тождество:

$$
A_{1 \rightarrow k}^{+}\left[\sigma_{k}^{x} \sigma_{k-1}^{x q^{2}} \ldots \sigma_{1}^{x q^{2(k-1)}}\right]=\left[\begin{array}{lll}
\sigma_{k}^{x q^{2(k-1)}} \sigma_{k-1}^{x q^{2(k-2)}} & \ldots \sigma_{2}^{x q^{2}} \sigma_{1}^{x}
\end{array}\right] A_{2 \rightarrow k+1}^{+},
$$

где $x$ - любой спектралъный параметр, $A_{1 \rightarrow k}^{+}$- симметризаторы, заданные в (7).

ДоказАтельство. Докажем уравнение (33) с помощью индукции. Пусть уравнение (33) справедливо для некоторого фиксированного $k$. Рассмотрим левую часть равенства (33) для случая $k+1$ :

$$
\begin{aligned}
& A_{1 \rightarrow k+1}^{+}\left[\sigma_{k+1}^{x} \sigma_{k}^{x q^{2}} \ldots \sigma_{1}^{x q^{2 k}}\right]=\left[e_{1}^{q^{-2}} \ldots e_{k}^{q^{-2 k}}\right] A_{1 \rightarrow k}^{+} \sigma_{k+1}^{x}\left[\sigma_{k}^{x q^{2}} \ldots \sigma_{1}^{x q^{2 k}}\right]= \\
& =\left[e_{1}^{q^{-2}} \ldots e_{k-1}^{q^{-2(k-1)}} e_{k}^{q^{-2 k}}\right] \sigma_{k+1}^{x}\left[\sigma_{k}^{x q^{2 k}} \sigma_{k-1}^{x q^{2(k-1)}} \ldots \sigma_{1}^{x q^{2}}\right] A_{2 \rightarrow k+1}^{+}=
\end{aligned}
$$

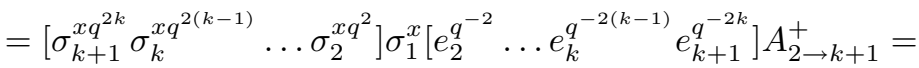

$$
\begin{aligned}
& =\left[\sigma_{k+1}^{x q^{2 k}} \ldots \sigma_{2}^{x q^{2}} \sigma_{1}^{x}\right] A_{2 \rightarrow k+2}^{+},
\end{aligned}
$$

где второе равенство в цепочке справедливо в силу предположения индукции (33), а третье - в силу уравнения Янга-Бакстера (5). Это соотношение эквивалентно (33), если в (33) сделать замену $k \rightarrow k+1$.

ПреДлОЖениЕ 1. Элементы трансфер-матричного типа $\tau^{(k)}(x)(28)$ удовлетворяют следующему тождеству:

$$
\tau^{(k)}(x) \tau^{(1)}\left(x q^{2 k}\right)=\phi_{k}^{\prime}(x) \tau^{(k+1)}(x)+\phi_{k}^{\prime \prime}(x) \tau^{(k, 1)}(x),
$$

где коэффициентные функиии имеют вид

$$
\begin{aligned}
\phi_{k}^{\prime}(x) & =\frac{\left(1-x^{2} q^{4 k} b^{-1}\right)\left(1-x^{2} q^{2(k-1)}\right)}{\left(1-x^{2} q^{2 k} b^{-1}\right)\left(1-x^{2} q^{4 k-2}\right)}, \\
\phi_{k}^{\prime \prime}(x) & =\frac{q^{k}\left(1-q^{-2 k}\right)}{\left(1-q^{-2(k+1)}\right)\left(1-q^{2}\right)^{k}} \frac{\left(1-x^{2} q^{2(k-1)} b^{-1}\right)\left(1-x^{2} q^{2(k-1)}\right)}{\left(1-x^{2} q^{2 k} b^{-1}\right)\left(1-x^{2} q^{4 k-2}\right)},
\end{aligned}
$$

a

$$
\tau^{(k, 1)}(x)=\operatorname{Tr}_{D(1 \rightarrow k+1)}\left(\left[\sigma_{1}^{q^{2}} \sigma_{2}^{q^{2}} \ldots \sigma_{k}^{q^{2}} A_{1 \rightarrow k}^{+}\right] \cdot y_{1 \rightarrow k+1}(x)\right)
$$

есть новый элемент трансфер-матричного типа.

ДокАЗАТЕЛЬСтво. Используя тождество (15) для $z=b / x$, мы выводим соотношение

$$
\begin{aligned}
\eta_{k}(x) \tau^{(1)}\left(x q^{2 k}\right) & =\eta_{k}(x) \operatorname{Tr}_{D(1)}\left(y_{1}^{x q^{2 k}}\right)= \\
& =\operatorname{Tr}_{D(k+1)}\left(\left[e_{k}^{x^{2} q^{2 k}} e_{k-1}^{x^{2} q^{2(k+1)}} \ldots e_{1}^{x^{2} q^{2(2 k-1)}}\right] y_{1}^{x q^{2 k}}\left[e_{1}^{\frac{b}{x^{2} q^{2(2 k-1)}}} \ldots e_{k}^{\frac{b}{x^{2} q^{2 k}}}\right]\right),
\end{aligned}
$$

где

$$
\eta_{k}(x)=\frac{\left(1-x^{2} q^{2(2 k-1)}\right)\left(1-x^{2} q^{2 k} b^{-1}\right)}{\left(1-x^{2} q^{2(k-1)}\right)\left(1-x^{2} q^{4 k} b^{-1}\right)} .
$$

Таким образом, мы имеем

$$
\eta_{k}(x) \tau^{(k)}(x) \tau^{(1)}\left(x q^{2 k}\right)=\operatorname{Tr}_{D(1 \rightarrow k+1)}\left(A_{1 \rightarrow k}^{+} \cdot y_{1 \rightarrow k+1}(x)\left[e_{1}^{\frac{b}{x^{2} q^{4 k-2}}} \ldots e_{k}^{\frac{b}{x^{2} q^{2 k}}}\right]\right) .
$$


Единичный элемент можно представить в виде линейной комбинации двух бакстеризованных элементов:

$$
1=e_{k}\left(q^{-2 k}\right)+\frac{\left(1-q^{-2 k}\right)}{\left(q^{2}-q^{-2 k}\right) \lambda} \sigma_{k}\left(q^{2}\right), \quad e_{k}\left(q^{-2 k}\right):=\frac{\sigma_{k}\left(q^{-2 k}\right)}{q-q^{-2 k-1}} .
$$

Принимая во внимание равенства (36), (33), мы записываем уравнение (35) в виде

$$
\begin{aligned}
& \eta_{k}(x) \tau^{(k)}(x) \tau^{(1)}\left(x q^{2 k}\right)=\operatorname{Tr}_{D(1 \rightarrow k+1)}\left(\left(A_{1 \rightarrow k+1}^{+}+\frac{\left(1-q^{-2 k}\right)}{\left(q^{2}-q^{-2 k}\right) \lambda} \sigma_{k}^{q^{2}} A_{1 \rightarrow k}^{+}\right) y_{1 \rightarrow k}(x) \times\right. \\
& \left.\quad \times\left[e_{k}^{x^{2} q^{2 k}} e_{k-1}^{x^{2} q^{2(k+1)}} \ldots e_{1}^{x^{2} q^{4 k-2}} y_{1}^{x q^{2 k}}\right] e_{1}^{\frac{b}{x^{2} q^{4 k-2}}} \ldots e_{k}^{\frac{b}{x^{2} q^{2 k}}}\right)= \\
& =\operatorname{Tr}_{D(1 \rightarrow k+1)}\left(A_{1 \rightarrow k+1}^{+} y_{1 \rightarrow k+1}(x) e_{1}^{\frac{b}{x^{2} q^{4 k-2}}} \ldots e_{k}^{\frac{b}{x^{2} q^{2 k}}}\right)+ \\
& \quad+\frac{\left(1-q^{-2 k}\right)}{\left(q^{2}-q^{-2 k}\right) \lambda} \operatorname{Tr}_{D(1 \rightarrow k+1)}\left(\bar{y}_{1 \rightarrow k}(x) \cdot\left[e_{k}^{x^{2} q^{4 k-2}} \ldots e_{1}^{x^{2} q^{2 k}} y_{1}^{x q^{2 k}}\right] \times\right. \\
& \left.\quad \times A_{2 \rightarrow k+1}^{+} e_{1}^{\frac{b}{x^{2} q^{4 k-2}}} \ldots e_{k}^{\frac{b}{x^{2} q^{2 k}}} \sigma_{k}^{q^{2}}\right) .
\end{aligned}
$$

Поскольку $\sigma_{k+1}^{q^{-2}} \sigma_{k+1}^{q^{2}}=0$, то можно вывести уравнение

$$
\sigma_{k+1}\left(q^{-2}\right) e_{k}(x) \sigma_{k+1}\left(q^{2}\right)=\frac{(1-x)}{\left(1-q^{2}\right)\left(q-q^{-1} x\right)} \sigma_{k+1}\left(q^{-2}\right) \sigma_{k}\left(q^{2}\right) \sigma_{k+1}\left(q^{2}\right),
$$

которое приводит к уравнению

$$
A_{2 \rightarrow k+1}^{+}\left[e^{\frac{b}{x^{2} q^{4 k-2}}} \ldots e_{k-1}^{\frac{b}{x^{2} q^{2 k+2}}} e_{k}^{\frac{b}{x^{2} q^{2 k}}} \sigma_{k}^{q^{2}}\right]=\frac{\left(-q^{-k-1}\right)\left(1-\frac{b}{x^{2} q^{2(k-1)}}\right)}{\left(1-q^{2}\right)^{k-1}\left(1-\frac{b}{x^{2} q^{4 k}}\right)} A_{2 \rightarrow k+1}^{+}\left[\sigma_{1}^{q^{2}} \ldots \sigma_{k}^{q^{2}}\right] .
$$

Мы также имеем тождество (см. последнее равенство в (8))

$$
A_{1 \rightarrow k+1}^{+}\left[e_{1}^{\frac{b}{x^{2} q^{4 k-2}}} \ldots e_{k}^{\frac{b}{x^{2} q^{2 k}}}\right]=A_{1 \rightarrow k+1}^{+} .
$$

Окончательно, используя (39) и (40), мы преобразуем (37) к виду

$$
\begin{array}{r}
\eta_{k}(x) \tau^{(k)}(x) \tau^{(1)}\left(x q^{2 k}\right)=\tau^{(k+1)}(x)+\frac{q^{-k-2}\left(1-q^{-2 k}\right)}{\left(1-q^{-2(k+1)}\right)\left(1-q^{2}\right)^{k}} \frac{\left(1-\frac{b}{x^{2} q^{2(k-1)}}\right)}{\left(1-\frac{b}{x^{2} q^{4 k}}\right)} \times \\
\times \operatorname{Tr}_{D(1 \rightarrow k+1)}\left(A_{1 \rightarrow k}^{+} \cdot y_{1 \rightarrow k+1}(x)\left[\sigma_{1}^{q^{2}} \ldots \sigma_{k-1}^{q^{2}} \sigma_{k}^{q^{2}}\right]\right),
\end{array}
$$

что эквивалентно уравнению (34).

\section{4. ФАКТОР ТЕМПЕРЛИ-ЛИБА ДЛЯ МОДЕЛИ ЦЕПОЧКИ ГЕККЕ}

Уравнения (34) не замкнуты относительно набора коммутирующих элементов трансфер-матричного типа $\tau^{(k)}(x)(31)$. Для того чтобы получить замкнутый набор соотношений, нам необходимо рассмотреть специальные факторы алгебры Гекке. В 
этом разделе мы рассматриваем фактор Темперли-Либа $H_{M+1}^{(\mathrm{TL})}$, который определяется наложением дополнительных связей

$$
\sigma_{i}\left(q^{2}\right) \sigma_{i \pm 1}\left(q^{4}\right) \sigma_{i}\left(q^{2}\right)=0
$$

на образующие $\sigma_{i} \in H_{M+1}(i=1, \ldots, M)$. Эти связи эквивалентны условию равенства нулю всех антисимметризаторов (7) третьего ранга: $A_{i \rightarrow i+2}^{-}=0$. Более того, оказывается, что эти связи фиксируют параметр $D^{(0)}$ (и, соответственно, параметр $b)$ :

$$
\begin{aligned}
0 & =\operatorname{Tr}_{D(i+1)}\left(\sigma_{i-1}\left(q^{2}\right) \sigma_{i}\left(q^{4}\right) \sigma_{i-1}\left(q^{2}\right)\right)=\sigma_{i-1}\left(q^{2}\right)\left(\operatorname{Tr}_{D(i+1)} \sigma_{i}\left(q^{4}\right)\right) \sigma_{i-1}\left(q^{2}\right)= \\
& =\sigma_{i-1}^{2}\left(q^{2}\right)\left(1-q^{4}\left(1-\lambda D^{(0)}\right)\right)=\sigma_{i-1}^{2}\left(q^{2}\right)\left(1-\frac{q^{4}}{b}\right) \Rightarrow \\
& \Rightarrow b=q^{4}, \quad D^{(0)}=\frac{1-q^{-4}}{\lambda} .
\end{aligned}
$$

Фактор Темперли-Либа $\widehat{H}_{M+1}^{(\mathrm{TL})}$ (ТЛ-фактор) аффинной алгебры Гекке определяется, кроме связей (41), еще и условием дополнительной связи на аффинную образующую $y_{1}$ алгебры $\widehat{H}_{M+1}$ :

$$
y_{1} \sigma_{1} y_{1} \sigma_{1}^{q^{2}}=\sigma_{1}^{q^{2}} y_{1} \sigma_{1} y_{1}=\Delta \sigma_{1}^{q^{2}},
$$

где $\Delta=\frac{\lambda q}{\left(1-q^{-4}\right)}\left(D^{(2)}-q\left(D^{(1)}\right)^{2}\right) \in \widehat{H}_{0}$ есть центральный элемент в $\widehat{H}_{M+1}$. Уравнение (43) при наличии отображения (14) требует, чтобы аффинная образующая $y_{1}$ подчинялась квадратичному характеристическому тождеству

$$
y_{1}^{2}-q D^{(1)} y_{1}=q^{-1} \Delta,
$$

после чего (43) переписывается в виде $\sigma_{1}^{q^{2}} y_{1} \sigma_{1}^{q^{2}}=q^{2}\left(q^{2}-1\right) D^{(1)} \sigma_{1}^{q^{2}}$. Таким образом, рассматриваемый фактор $\widehat{H}_{M+1}^{(\mathrm{TL})}$ аффинной алгебры Гекке совпадает с однограничной алгеброй Темперли-Либа, или blob-алгеброй [14]. Связь (43) также ведет к связям

$$
y_{1}^{z q^{-2}} e_{1}^{z^{2} q^{-2}} y_{1}^{z} \sigma_{1}^{q^{2}}=\sigma_{1}^{q^{2}} y_{1}^{z} e_{1}^{z^{2} q^{-2}} y_{1}^{z q^{-2}}=: \Delta^{(0)}(z) \sigma_{1}^{q^{2}},
$$

где $y_{1}^{z} \in \widehat{H}_{M+1}$ - любое локальное решение уравнения отражения (11) для $n=1$, а $\Delta^{(0)}(z)$ - центральный элемент в $\widehat{H}_{M+1}$.

ПрЕДЛОЖЕНИЕ 2. В случае ТЛ-фактора для открытой цепочки Гекке (т.е. когда $\left.\left\{\sigma_{i}, y_{1}\right\} \in \widehat{H}_{M+1}^{(\mathrm{TL})}\right)$ функииональные соотношения (34) замыкаются относительно семейства элементов трансфер-матричного типа $\tau^{(k)}(x)$ :

$$
\tau^{(k)}(x) \tau^{(1)}\left(x q^{2 k}\right)=\phi_{k}^{\prime}(x) \tau^{(k+1)}(x)+\phi_{k}^{\prime \prime \prime}(x) \Delta^{(0)}\left(x q^{2 k}\right) \tau^{(k-1)}(x),
$$

где $\tau^{(0)}(x):=1 u$

$$
\begin{aligned}
\phi_{k}^{\prime}(x) & :=\frac{\left(1-x^{2} q^{2(k-1)}\right)\left(1-x^{2} q^{4 k-4}\right)}{\left(1-x^{2} q^{2 k-4}\right)\left(1-x^{2} q^{4 k-2}\right)} \\
\phi_{k}^{\prime \prime \prime}(x) & :=\frac{1}{\left(-q^{2}\right)} \frac{\left(1-x^{2} q^{2 k-6}\right)\left(1-x^{2} q^{4 k-4}\right)}{\left(1-x^{2} q^{2 k-4}\right)\left(1-x^{2} q^{4 k-2}\right)}
\end{aligned}
$$


ДоказАтельство. Запишем (34) в виде

$$
\begin{aligned}
\tau^{(k)}(x) \tau^{(1)}\left(x q^{2 k}\right)=\phi_{k}^{\prime}(x) \tau^{(k+1)}(x)+\phi_{k}^{\prime \prime}(x) \Delta^{(0)}\left(x q^{2 k}\right) \times & \\
\times & \operatorname{Tr}_{D(1 \rightarrow k+1)}\left(A_{1 \rightarrow k}^{+} \cdot y_{1 \rightarrow k-1}(x) \cdot\left[e_{k-1}^{x^{2} q^{2(k-1)}} \ldots e_{1}^{x^{2} q^{4 k-6}}\right] \times\right. \\
& \left.\times\left[e_{k}^{x^{2} q^{2 k}} \ldots e_{2}^{x^{2} q^{4(k-1)}}\right]\left[\sigma_{1}^{q^{2}} \ldots \sigma_{k}^{q^{2}}\right]\right) .
\end{aligned}
$$

Связи (41), которые определяют ТЛ-фактор алгебры Гекке, ведут к следующим тождествам: для любого $x$

$$
\begin{aligned}
\sigma_{i}\left(q^{2}\right) \sigma_{i \pm 1}(x) \sigma_{i}\left(q^{2}\right) & =\lambda q^{3}\left(1-q^{-4} x\right) \sigma_{i}\left(q^{2}\right), \\
e_{i}(x) e_{i \pm 1}\left(x q^{2}\right) \sigma_{i}\left(q^{2}\right) & =-\xi(x) \sigma_{i \pm 1}\left(q^{2}\right) \sigma_{i}\left(q^{2}\right)
\end{aligned}
$$

где $\xi(x)=\frac{\left(1-x q^{2}\right)}{q^{2} \lambda(1-x)}$. Мы используем эти тождества для того, чтобы упростить (46) с помощью следующего соотношения:

$$
\begin{aligned}
& {\left[e_{k-1}^{x^{2} q^{2(k-1)}} \ldots e_{1}^{x^{2} q^{4 k-6}}\right]\left[e_{k}^{x^{2} q^{2 k}} \ldots e_{2}^{x^{2} q^{4(k-1)}}\right]\left[\sigma_{1}^{q^{2}} \ldots \sigma_{k-1}^{q^{2}} \sigma_{k}^{q^{2}}\right]=}
\end{aligned}
$$

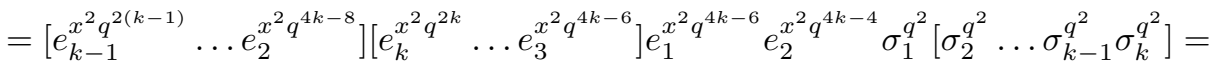

$$
\begin{aligned}
& =\left(-\xi\left(x^{2} q^{4 k-6}\right)\right)\left[e_{k-1}^{x^{2} q^{2(k-1)}} \ldots e_{2}^{x^{2} q^{4 k-8}}\right]\left[e_{k}^{x^{2} q^{2 k}} \ldots e_{3}^{x^{2} q^{4 k-6}}\right] \sigma_{2}^{q^{2}} \sigma_{1}^{q^{2}}\left[\sigma_{2}^{q^{2}} \ldots \sigma_{k-1}^{q^{2}} \sigma_{k}^{q^{2}}\right]=\cdots \\
& \cdots=\prod_{n=1}^{k-1}\left(-\xi\left(x^{2} q^{4 k-4-2 n}\right)\right)\left[\sigma_{k}^{q^{2}} \sigma_{k-1}^{q^{2}} \ldots \sigma_{2}^{q^{2}} \sigma_{1}^{q^{2}} \sigma_{2}^{q^{2}} \ldots \sigma_{k-1}^{q^{2}} \sigma_{k}^{q^{2}}\right]= \\
& =(\lambda q)^{2(k-1)} \prod_{n=1}^{k-1}\left(-\xi\left(x^{2} q^{4 k-4-2 n}\right)\right) \sigma_{k}^{q^{2}}=(-\lambda)^{k-1} \frac{1-x^{2} q^{4(k-1)}}{1-x^{2} q^{2(k-1)}} \sigma_{k}^{q^{2}} .
\end{aligned}
$$

Принимая во внимание это соотношение и тождества

$$
\operatorname{Tr}_{D(k+1)}\left(\sigma_{k}^{q^{2}}\right)=1-\frac{q^{2}}{b}, \quad \operatorname{Tr}_{D(k)} A_{1 \rightarrow k}^{+}=\frac{1-q^{-2(k-1)} / b}{q-q^{-2 k+1}} A_{1 \rightarrow k-1}^{+},
$$

которые следуют из определений (7), (14), мы записываем (46) для $b=q^{4}$ в виде (45).

Уравнения (45) замкнуты относительно набора коммутирующих элементов $\tau^{(k)}(x)$. Заметим, что уравнения (45) могут быть использованы для определения элементов $\tau^{(k)}(x)$ в случае отрицательных целых $k$.

\section{T- $Q$ УРАВНЕНИЕ БАКСТЕРА ДЛЯ ОТКРЫТЫХ ЦЕПОЧЕК ТЛ-ТИПА}

Сделаем теперь замену переменных $z=x q^{2 k}$ в (45) и положим

$$
Q^{(k)}(z):=\left(1-z^{2} q^{-2(k+2)}\right) \tau^{(k)}\left(z q^{-2 k}\right) .
$$

В результате уравнения (45) записываются в виде

$$
\frac{\left(1-z^{2} q^{-2}\right)}{\left(1-z^{2} q^{-4}\right)} Q^{(k)}(z) \tau^{(1)}(z)=Q^{(k+1)}\left(z q^{2}\right)-\frac{\Delta^{(0)}(z)}{q^{2}} Q^{(k-1)}\left(z q^{-2}\right),
$$


где $\tau^{(1)}(z)$ и $\Delta^{(0)}(z)$ определяются в $(32)$ и $(44)$, соответственно. Введем производящую функцию $Q(z, w)$ элементов $Q^{(k)}(z)$ :

$$
Q(z, w):=\sum_{k=-\infty}^{\infty} w^{k} Q^{(k)}(z)
$$

Тогда уравнение (47) представляется в виде $T-Q$ уравнения Бакстера

$$
\frac{\left(1-z^{2} q^{-2}\right)}{\left(1-z^{2} q^{-4}\right)} Q(z, w) \tau^{(1)}(z)=\frac{1}{w} Q\left(z q^{2}, w\right)-\frac{\Delta^{(0)}(z) w}{q^{2}} Q\left(z q^{-2}, w\right) .
$$

Напомним, что нашей целью является поиск спектра оператора трансфер-матричного типа $\tau^{(1)}(z)$ для некоторой фиксированной детерминантной функции $\Delta^{(0)}(z)$. Мы фиксируем явный вид детерминанта $\Delta^{(0)}(z)(44)$, рассматривая специальную модель открытой цепочки с $N$ узлами. В этом случае $\Delta^{(0)}(z)=: \Delta_{N}^{(0)}(z)$ определяется соотношением

$$
\Delta_{N}^{(0)}(z) \sigma_{N+1}^{q^{2}}=y_{N+1}^{z q^{-2}} e_{N+1}^{z^{2} q^{-2}} y_{N+1}^{z} \sigma_{N+1}^{q^{2}}
$$

где элемент монодромии $y_{N+1}^{z}$ представляет собой перенормированную версию элемента (13) и задается формулой

$$
y_{N+1}^{z}=e_{N}^{+}(z) y_{N}^{z} e_{N}^{+}(z)=\cdots=e_{N}^{+}(z) \ldots e_{1}^{+}(z) y_{1}^{z} e_{1}^{+}(z) \ldots e_{N}^{+}(z) .
$$

Пусть $y_{1}^{z}$ с точностью до умножения на некоторую несущественную скалярную функцию от $z$ представляется в виде полинома по $z$. Такие решения $y_{1}^{z}$ уравнения отражения были предложены в [2]. Мы заметим, что для случая, когда $y_{1}^{z}$ есть полином по $z$ порядка $K$, элемент трансфер-матричного типа записывается в виде

$$
\tau_{N}^{(1)}(z)=\operatorname{Tr}_{D(N+1)}\left(y_{N+1}^{z}\right)=\frac{1}{\left(q-z q^{-1}\right)^{2 N}} \tau_{N}(z),
$$

где элемент $\tau_{N}(z)(20)$ есть полином по $z$ порядка $2 N+K$. Тогда из (49) мы получаем

$$
\begin{aligned}
\Delta_{N}^{(0)}(z) \sigma_{N+1}^{q^{2}} & =\left(e_{N}^{z q^{-2}} y_{N}^{z q^{-2}} e_{N}^{z q^{-2}}\right) e_{N+1}^{z^{2} q^{-2}}\left(e_{N}^{z} y_{N}^{z} e_{N}^{z}\right) \sigma_{N+1}^{q^{2}}= \\
& =e_{N}^{z q^{-2}} e_{N+1}^{z} y_{N}^{z q^{-2}} e_{N}^{z^{2} q^{-2}} y_{N}^{z}\left(e_{N+1}^{z q^{-2}} e_{N}^{z} \sigma_{N+1}^{q^{2}}\right)= \\
& =-\xi\left(z q^{-2}\right) e_{N}^{z q^{-2}} e_{N+1}^{z}\left(y_{N}^{z q^{-2}} e_{N}^{z^{2} q^{-2}} y_{N}^{z} \sigma_{N}^{q^{2}}\right) \sigma_{N+1}^{q^{2}}= \\
& =\left(\xi\left(z q^{-2}\right)\right)^{2} \sigma_{N+1}^{q^{2}}\left[\Delta_{N-1}^{(0)}(z) \sigma_{N}^{q^{2}}\right] \sigma_{N+1}^{q^{2}}= \\
& =\cdots=\left(\xi\left(z q^{-2}\right)\right)^{2 N} \sigma_{N+1}^{q^{2}} \ldots \sigma_{2}^{q^{2}}\left[y_{1}^{z} e_{1}^{z^{2} q^{-2}} y_{1}^{z q^{-2}} \sigma_{1}^{q^{2}}\right] \sigma_{2}^{q^{2}} \ldots \sigma_{N+1}^{q^{2}}= \\
& =\Delta_{0}^{(0)}(z)\left(\xi\left(z q^{-2}\right) \lambda q\right)^{2 N} \sigma_{N+1}^{q^{2}},
\end{aligned}
$$

откуда

$$
\Delta_{N}^{(0)}(z)=\Delta_{0}^{(0)}(z)\left(\xi\left(z q^{-2}\right) \lambda q\right)^{2 N}=\Delta_{0}^{(0)}(z)\left(\frac{1-z}{q-z q^{-1}}\right)^{2 N}
$$


Мы выбираем тривиальное граничное условие $y_{1}^{z}(z)=1$, соответствующее модели открытой цепочки со свободными концами, которая описывается гамильтонианом $\mathcal{H}_{N}^{(\text {free) }}(23)$. В этом случае начальный детерминант $\Delta_{0}^{(0)}(z)$ фиксируется соотношением

$$
\Delta_{0}^{(0)}(z) \sigma_{1}^{q^{2}}=e_{1}^{z^{2} q^{-2}} \sigma_{1}^{q^{2}}=-q^{-2} \frac{\left(1-z^{2}\right)}{\left(1-z^{2} q^{-4}\right)} \sigma_{1}^{q^{2}} .
$$

Таким образом, функциональные уравнения (47) представляются как

$$
\begin{aligned}
& \frac{1-z^{2} q^{-2}}{1-z^{2} q^{-4}} Q^{(k)}(z) \frac{T_{N}^{(1)}(z)}{\left(q-z q^{-1}\right)^{2 N}}=Q^{(k+1)}\left(z q^{2}\right)+ \\
& \quad+q^{-4} \frac{1-z^{2}}{1-z^{2} q^{-4}}\left(\frac{1-z}{q-z q^{-1}}\right)^{2 N} Q^{(k-1)}\left(z q^{-2}\right),
\end{aligned}
$$

где мы подставили $\tau_{N}^{(1)}(z)=T_{N}^{(1)}(z) /\left(q-z q^{-1}\right)^{2 N}$, а $T_{N}^{(1)}(z)=\left.\tau_{N}(z)\right|_{y_{1}^{z}=1}$ есть полином по $z$ порядка $2 N$, который рассматривался в разделе 2 , см. формулу (21).

Уравнения (48) и (53) могут быть использованы стандартным образом для вывода уравнений аналитического анзаца Бете, которые содержат информацию о спектре операторов трансфер-матричного типа $\tau^{(1)}(z)$ и, в частности, о спектре гамильтонианов (18).

\section{6. ЗАКЛЮЧЕНИЕ}

Обсудим кратко взаимосвязи между алгебраическим подходом, изложенным выше, и стандартной техникой, развитой в [10] для исследования спиновых систем на открытых цепочках и основанной на матричном уравнении отражения.

Рассмотрим $R$-матричное представление $\rho_{R}$ аффинной алгебры Гекке $\widehat{H}_{M+1}$ :

$$
\begin{aligned}
& \rho_{R}\left(\sigma_{i}\right)=\widehat{R}_{i i+1}:=I^{\otimes(i-1)} \otimes \widehat{R} \otimes I^{\otimes(M-i)}, \\
& \rho_{R}\left(y_{1}\right)=S\left(L_{1}^{-}\right) L_{1}^{+}:=\left(1 / L^{-}\right) L^{+} \otimes I^{\otimes M} .
\end{aligned}
$$

Здесь $I$ есть единичная $((n+m) \times(n+m))$-матрица; $\widehat{R} \in \operatorname{End}\left(V_{n+m}^{\otimes 2}\right)$ - фундаментальная $R$-матрица для $U_{q}(g l(n \mid m))$, записанная в форме элемента группы кос [5], [11] (стандартная форма приведена в [15]):

$$
\begin{aligned}
\widehat{R} & =\sum_{i}(-1)^{[i]} q^{1-2[i]} e_{i i} \otimes e_{i i}+\sum_{i \neq j}(-1)^{[i][j]} e_{i j} \otimes e_{j i}+\lambda \sum_{j>i} e_{i i} \otimes e_{j j}, \\
\widehat{R}^{2} & =\lambda \widehat{R}+1, \quad(-1)^{(1)(2)} \widehat{R}_{12}=\widehat{R}_{12}(-1)^{(1)(2)}, \quad \lim _{q \rightarrow 1}\left(\widehat{R}_{12}\right)=\mathcal{P}_{12},
\end{aligned}
$$

где $\lambda=q-q^{-1}, e_{i j}$ - матричные единицы, $[i]=0,1(\bmod 2)$ обозначает грассманову четность соответствующих компонент супервекторов в пространстве $V_{n+m}$, $\mathcal{P}_{12}:=(-1)^{(1)(2)} P_{12}$ - матрица суперперестановки $\left(P_{12}\right.$ - матрица перестановки). Мы использовали компактные матричные обозначения, например, $\left((-1)^{(1)(2)}\right)_{j_{1} j_{2}}^{i_{1} i_{2}}=$ $(-1)^{\left[i_{1}\right]\left[i_{2}\right]} \delta_{j_{1}}^{i_{1}} \delta_{j_{2}}^{i_{2}}$. Операторнозначные матрицы $L^{+}\left(L^{-}\right) \in \operatorname{Mat}(n+m)$ обратимы, верхнетреугольны (нижнетреугольны) и удовлетворяют равенствам

$$
\begin{aligned}
& \widehat{R}_{12} L_{2}^{ \pm}(-1)^{(1)(2)} L_{1}^{ \pm}=L_{2}^{ \pm}(-1)^{(1)(2)} L_{1}^{ \pm} \widehat{R}_{12}, \\
& \widehat{R}_{12} L_{2}^{+}(-1)^{(1)(2)} L_{1}^{-}=L_{2}^{-}(-1)^{(1)(2)} L_{1}^{+} \widehat{R}_{12} .
\end{aligned}
$$


Мы приписываем элементам $\left(L^{ \pm}\right)_{j}^{i}$ градуировку $([i]+[j])$. В соответствии с подходом работы [4] $L^{ \pm}$являются матрицами генераторов картановского типа для $U_{q}(g l(n \mid m))$. Заметим, что в представлении $\rho_{R}(54)$ решение (12) принимает вид

$$
\rho_{R}\left(y_{1}(x)\right)=\frac{1}{L_{1}^{+}-\xi x^{-1} L_{1}^{-}}\left(L_{1}^{+}-\xi x L_{1}^{-}\right)=: K_{1}(x),
$$

и можно сразу проверить, что (56) есть решение уравнения отражения (11), записанного в $R$-матричной форме:

$$
\widehat{R}_{12}(x / z) K_{1}(x) \widehat{R}_{12}(x z) K_{1}(z)=K_{1}(z) \widehat{R}_{12}(x z) K_{1}(x) \widehat{R}_{12}(x / z),
$$

где

$$
\widehat{R}_{n n+1}(x):=\widehat{R}_{n n+1}-x \widehat{R}_{n n+1}^{-1}=\rho_{R}\left(\sigma_{n}\right)
$$

является $R$-матричным образом бакстеризованного элемента (4). Для того чтобы проверить (57), необходимо учесть представление $K(x)(56)$ в факторизованном виде $L^{-1}(\xi / x) L(\xi x)$, где операторнозначная матрица

$$
L(x)=L^{+}-x L^{-}
$$

определяет evaluation-представление [12] для аффинной квантовой супералгебры $U_{q}(\hat{g l}(n \mid m))$ и удовлетворяет сплетающим соотношениям

$$
\widehat{R}_{12}(x) L_{2}(x y)(-1)^{(1)(2)} L_{1}(y)=L_{2}(y)(-1)^{(1)(2)} L_{1}(x y) \widehat{R}_{12}(x) .
$$

Отметим, что все факторы типа $(-1)^{(1)(2)}$ пропали в уравнении отражения (57). Коумножения для квантовых супералгебр с определяющими соотношениями (55) и (59) имеют обычный вид $\Delta L^{ \pm}=L^{ \pm} \otimes L^{ \pm}$и $\tilde{\Delta} L(x)=L(x) \otimes L(x)$, где $\otimes$ - градуированное тензорное произведение, т.е. $(a \otimes b)(c \otimes d)=(-1)^{[b][c]}(a c \otimes b d)$.

Отметим, что решения уравнения отражения типа (56) были названы в [16] решениями, которые допускают "регулярную факторизацию". Задавая матрицу $L(x)$, которая удовлетворяет (59), мы с помощью стандартного метода квантовой обратной задачи можем построить операторнозначную матрицу монодромии для цепочки с $(N+1)$ узлами:

$$
\mathcal{T}_{a}\left(\xi, \xi_{1}, \ldots, \xi_{N} \mid x\right)=L_{a}(\xi x) \otimes L_{a}\left(\xi_{1} x\right) \otimes \cdots \otimes L_{a}\left(\xi_{N} x\right),
$$

где индекс $a$ обозначает номер вспомогательного матричного пространства, $\otimes-$ градуированное тензорное произведение операторных пространств. Матрица монодромии (60) удовлетворяет сплетающим соотношениям (59)

$$
\begin{aligned}
& \widehat{R}_{12}(x) \mathcal{T}_{2}\left(\xi, \xi_{1}, \ldots, \xi_{N} \mid x y\right)(-1)^{(1)(2)} \mathcal{T}_{1}\left(\xi, \xi_{1}, \ldots, \xi_{N} \mid y\right)= \\
& \quad=\mathcal{T}_{2}\left(\xi, \xi_{1}, \ldots, \xi_{N} \mid y\right)(-1)^{(1)(2)} \mathcal{T}_{1}\left(\xi, \xi_{1}, \ldots, \xi_{N} \mid x y\right) \widehat{R}_{12}(x)
\end{aligned}
$$

Далее мы строим двухрядную матрицу монодромии Склянина

$$
\mathcal{K}_{a}\left(\xi, \xi_{1}, \ldots, \xi_{N} \mid x\right)=\mathcal{T}_{a}^{-1}\left(\xi, \xi_{1}, \ldots, \xi_{N} \mid x^{-1}\right) \cdot \mathcal{T}_{a}\left(\xi, \xi_{1}, \ldots, \xi_{N} \mid x\right),
$$


которая очевидно удовлетворяет уравнению отражения (57). Соответствующая трансфер-матрица Склянина задается квантовым суперследом

$$
\tau_{N}\left(\xi, \xi_{1}, \ldots, \xi_{N} \mid x\right)=\operatorname{Tr}_{a}\left(D_{a} \mathcal{K}_{a}\left(\xi, \xi_{1}, \ldots, \xi_{N} \mid x\right)\right)
$$

где $D_{j}^{i}=(-1)^{[i]} q^{2 m+(-1)^{[i]}(2 i-2 m-1)} \delta_{j}^{i}$ - матрица квантового суперследа (для случая квантовой супералгебры $U_{q}(g l(n \mid m))$ [11]. Можно проверить, что эта матрица является константным решением сопряженного уравнения отражения. Примем во внимание тот факт, что матрица монодромии (60) удовлетворяет сплетающим соотношениям (59); тогда можно использовать матричное представление, в котором образ (60) есть

$$
\begin{array}{r}
\mathcal{T}_{a}\left(\xi, \xi_{1}, \ldots, \xi_{N} \mid x\right) \rightarrow(-1)^{((1)+\cdots+(N))(a)} L_{a}(\xi x) P_{1 a} \widehat{R}_{1 a}\left(\xi_{1} x\right) \ldots P_{N a} \widehat{R}_{N a}\left(\xi_{N} x\right)= \\
=\mathcal{P}_{N a} \mathcal{P}_{N-1 N} \ldots \mathcal{P}_{23} \mathcal{P}_{12} L_{1}(\xi x) \widehat{R}_{12}\left(\xi_{1} x\right) \ldots \widehat{R}_{N-1 N}\left(\xi_{N-1} x\right) \widehat{R}_{N a}\left(\xi_{N} x\right),
\end{array}
$$

где мы применили матричный гомоморфизм ко всем факторам в правой части (60) за исключением первого фактора, а $1,2, \ldots, N$ обозначают номера матричных пространств, которые заменяют операторные пространства. Затем для матрицы монодромии Склянина (61) мы получаем представление

$$
\begin{aligned}
\mathcal{K}_{a}\left(\xi, \xi_{1}, \ldots, \xi_{N} \mid x\right) \rightarrow \widehat{R}_{N a}^{-1}\left(\frac{\xi_{N}}{x}\right) \widehat{R}_{N-1 N}^{-1}\left(\frac{\xi_{N-1}}{x}\right) \ldots \widehat{R}_{12}^{-1}\left(\frac{\xi_{1}}{x}\right) \times \\
\times \frac{1}{L_{1}(\xi / x)} L_{1}(\xi x) \widehat{R}_{12}\left(\xi_{1} x\right) \ldots \widehat{R}_{N-1 N}\left(\xi_{N-1} x\right) \widehat{R}_{N a}\left(\xi_{N} x\right)= \\
=\rho_{R}\left(e_{N}^{+}\left(\frac{x}{\xi_{N}}\right) e_{N-1}^{+}\left(\frac{x}{\xi_{N-1}}\right) \ldots e_{1}^{+}\left(\frac{x}{\xi_{1}}\right) \times\right. \\
\left.\times \frac{y_{1}-\xi x}{y_{1}-\xi / x} e_{1}^{+}\left(\xi_{1} x\right) \ldots e_{N-1}^{+}\left(\xi_{N-1} x\right) e_{N}^{+}\left(\xi_{N} x\right)\right)
\end{aligned}
$$

где мы учли тождество $\widehat{R}\left(x^{-1}\right) \widehat{R}(x)=\left(q-x q^{-1}\right)\left(q-x^{-1} q^{-1}\right)$, положили $a=N+$ 1 и использовали обозначения (6), (54). Ясно, что (63) есть $R$-матричный образ элемента (50) для $x=z, \xi_{i}=1$ и $y_{1}^{x}=\left(y_{1}-\xi x\right) /\left(y_{1}-\xi / x\right)$. Квантовый след (62) от (63) совпадает с $R$-матричным образом $\rho_{R}$ элемента трансфер-матричного типа (51).

Итак, мы продемонстрировали, что интегрируемые модели, основанные на рассмотрении матриц монодромии (61), и модели, основанные на рассмотрении элементов монодромии (50), в общем случае различны и совпадают друг с другом только в случае их $R$-матричных проекций. Такое отождествление проясняется формулой (63).

Благодарности. Автор благодарен П.П. Кулишу за полезные обсуждения и комментарии, а также А.Ф. Оськину, С. З. Пакуляку и П. Н. Пятову за обсуждение результатов, изложенных в разделе 2. Данная работа поддержана INTAS (грант № 03-51-3350) и РФФИ (грант № 05-01-01086-а). 


\section{Список литературы}

[1] A. P. Isaev, O. V. Ogievetsky, Nucl. Phys. B, 760 (2007), 167; math-ph/0510078.

[2] D. Levi, P. Martin, J. Phys. A, 27 (1994), L521; P. P. Kulish, A. I. Mudrov, Lett. Math. Phys., 75:2 (2006), 151; math.QA/0508289.

[3] M. Jimbo, Lett. Math. Phys., 11 (1986), 247.

[4] Н. Ю. Решетихин, Л. А. Тахтаджян, Л. Д. Фаддеев, Алгебра и анализ, 1:1 (1989), 178.

[5] A. P. Isaev, ЭЧАЯ, 26 (1995), 1204.

[6] P.P. Kulish, N. Yu. Reshetikhin, E.K. Sklyanin, Lett. Math. Phys., 5:5 (1981), 393; P. P. Kulish, E. K. Sklyanin, "Quantum spectral transform method. Recent developments", Integrable Quantum Field Theories, Proc. Symp. (Tvärminne, Finland, 1981), Lecture Notes in Phys., 151, eds. J. Hietarinta, C. Montonen, Springer, Berlin, 1982, 61.

[7] L. Mezinchescu, R. Nepomechie, J. Phys. A, 25 (1992), 2533.

[8] Y.K. Zhou, Nucl. Phys. B, 458 (1996), 504; hep-th/9510095; W. L. Yang, R. I. Nepomechie, Y.Z. Zhang, Phys. Lett. B, 633 (2006), 664; hep-th/0511134.

[9] P. P. Kulish, J. Phys. A, 36 (2003), L489.

[10] E. K. Sklyanin, J. Phys. A, 21 (1988), 2375.

[11] A.P. Isaev, Quantum groups and Yang-Baxter equations, Preprint MPIM (Bonn), MPIM2004-132, 2004; http://www.mpim-bonn.mpg.de/Research/MPIM+Preprint+Series.

[12] V. Chari, A. Pressley, A Guide to Quantum Groups, Cambrige Univ. Press, 1994.

[13] A. P. Isaev, O. V. Ogievetsky, Czech. J. Physics, 55:11 (2005), 1433; O. V. Ogievetsky, P. N. Pyatov, "Lecture on Hecke algebras", Symmetries and Integrable Systems, Proc. Int. School (Dubna, Russia, 1999), ed. A. N. Sissakian, JINR, Dubna, 2000, 39; Preprint MPIM (Bonn), MPIM2001-40; http://www . mpim-bonn.mpg.de/Research/MPIM+Preprint+Series.

[14] D. Levy, Phys. Rev. Lett., 67 (1991), 1971; Int. J. Mod. Phys. A, 6 (1991), 5127; P. P. Martin, H. Saleur, Lett. Math. Phys., 30:3 (1994), 189; hep-th/9302094; P. P. Martin, D. Woodcock, J. Algebra, 225:2 (2000), 957.

[15] Е. В. Дамаскинский, П.П. Кулиш, М. А. Соколов, Записки науч. сем. ПОМИ, 224 (1995), 155; q-alg/9505001.

[16] H. Frahm, N. Slavnov, J. Phys. A, 32 (1999), 1547. 